\title{
Dextrose prolotherapy for rotator cuff lesions: the challenges and the future
}

Hong Kong Med J 2018;24:538-9

DOI: $10.12809 / \mathrm{hkmj} 187528$

To the Editor-We appreciated the letter by Dr Reza Ganji ${ }^{1}$ about chronic rotator cuff lesions (RCLs), in which he identifies prolotherapy as a potential treatment, noted a potential mechanism of action, reviewed three studies, and concluded that prolotherapy is promising but not ready for general use due to insufficient evidence. Although we agree with some elements of his letter, each of his assertions requires either additional information or respectful correction to better serve both physicians and their patients.

Dextrose prolotherapy is an evidence-based injection therapy for chronic musculoskeletal pain. ${ }^{2}$ It has been used for approximately 100 years and its modern applications can be traced to the 1950 s when prolotherapy injection protocols were formalised by George Hackett, ${ }^{3}$ a general surgeon in the United States, based on his clinical experience of over 30 years. Although a variety of injectants have been used, hypertonic dextrose is the most commonly used and best studied. The number of papers devoted to prolotherapy has steadily grown over the past 20 years, as has the number of countries from which these studies originate. This suggests increased clinical use, although whether this is true and to what extent are unknown.

The fundamental innovation brought by prolotherapy to the treatment of chronic musculoskeletal pain is its potential therapeutic effects on multiple pain generators within and around joints. Prolotherapy is unique in its nonsurgical targeting of multiple pathological structures and its mechanism of action is likely multifactorial. Hypertonic dextrose is thought to facilitate healing and subsequent pain control through tissue change or proliferation, potentially mediated by an inflammatory mechanism, thus improving joint stability and biomechanics, and ultimately decreasing pain. ${ }^{4}$ However, other mechanisms have been proposed and are evidence-based, including the direct sensorineural effect of dextrose on pain control. ${ }^{5,6}$ This potential effect has been suggested in clinical studies of epidural injection of dextrose for chronic recalcitrant low back pain, ${ }^{7}$ and dextrose injections for carpal tunnel syndrome and Achilles tendinosis. ${ }^{8,9}$

Studies have shown that prolotherapy is efficacious in the management of knee osteoarthritis and tendinopathy. ${ }^{10,11}$ We agree that the evidence to support its use in the context of RCLs is less robust and that more and better evidence is needed to determine its precise contribution to care of RCLs. However, in clinical life, no treatment works for every patient. We suggest that ample evidence exists for clinicians to add prolotherapy to their therapeutic armamentarium for carefully selected patients. As Dr Ganji notes:

1. Prolotherapy in the described studies is safe, well-tolerated, and satisfactory to patients. This is impressive given that injection-based care is inherently mildly traumatic and uncomfortable. Patients appear to be voting with their feet.

2. Prolotherapy is reported to improve chronic rotator cuff pain refractory to other modalities. Pain is the main reason patients come to see physicians.

3. Prolotherapy is reported to improve function, an essential outcome that is both related to and separate from pain.

In the complex work of treating chronic pain in RCLs, we believe prolotherapy has a more robust place in care than that suggested by Dr Ganji. We advocate conservative management as initial treatment for RCLs, including activity modification, anti-inflammatory medication, and physical therapy. Surgery is typically reserved for young athletes, or patients with full thickness tears or those who have failed conservative treatment. Injection therapy is generally regarded as adjuvant therapy to nonoperative treatment, among which corticosteroid is commonly studied and is well-known for its short-term pain relief, but its detrimental effect on cartilage that should not be overlooked. We are using fewer corticosteroid injections and we consider prolotherapy to be a part of non-surgical care for patients with RCLs who are refractory to the more conservative steps.

We agree with Dr Ganji's assertion that more high quality clinical trials are needed to determine specific elements of the efficacy of prolotherapy in RCLs. We suggest consideration of some important elements in trial design. First, an accurate diagnosis of pathology in RCLs should be made. Prolotherapy is effective for tendinopathies ${ }^{10}$ but may be less so for pathologies such as impingement and SLAP lesions. Second, it is reasonable to begin prolotherapy for RCLs using a whole joint approach with the clinical standard of $15 \%$ dextrose for extra-articular injections and $25 \%$ for intra-articular injections. ${ }^{12,13}$ However, in time, formal assessment of different 
concentrations will be needed. If the aetiology of pain is mostly neuropathic, the use of $5 \%$ dextrose may be preferred. ${ }^{8}$ Third, although there is no consensus on the optimal injection frequency, again it is reasonable to use the clinical standard of 3 to 5 injection sets 4 weeks apart followed by a booster session 8 weeks later. ${ }^{12,13}$ Fourth, the choice of control therapy should be made with caution. Although many clinical trialists and granting agencies consider a blinded placebo injection to be necessary, normal saline injections are now understood to be active therapy. ${ }^{14}$ Even sham needling has been shown to provide therapeutic effects in pain control. ${ }^{15}$ Therefore, non-injection control groups, such as standard-of-care therapy, while having some disadvantages, may be appropriate in some trials. Finally, validated and guideline-recommended selfreported and objectively assessed patient outcomes specific to shoulder injury should be used to assess the effects of prolotherapy. ${ }^{16}$

In conclusion, we agree that dextrose prolotherapy is not yet the first-line treatment for RCLs but clinicians can feel comfortable with its use in carefully selected patients who are refractory to other conservative treatments.

\section{Declaration}

All authors have disclosed no conflicts of interest. All authors had full access to the data, contributed to the study, approved the final version for publication, and take responsibility for its accuracy and integrity.

\section{${ }^{1}$ Regina WS Sit *, MB, BS, FHKAM (Family Medicine)}

${ }^{2}$ David Rabago, MD

1 The School of Public Health and Primary Care, The Chinese University of Hong Kong, Shatin, Hong Kong

${ }^{2}$ Department of Family Medicine, University of Wisconsin School of Medicine and Public Health, Madison, Wisconsin, United States

* Corresponding author: reginasit@cuhk.edu.hk

\section{References}

1. Ganji R. Dextrose prolotherapy for improvement of rotator cuff lesions: ready for clinical use? Hong Kong Med J 2018;24:429-30.

2. Reeves KD, Sit RW, Rabago DP. Dextrose prolotherapy: a narrative review of basic science, clinical research, and best treatment recommendations. Phys Med Rehabil Clin 2016;27:783-823.

3. Hackett GS. Ligament and Tendon Relaxation (Skeletal disability). Springfield, IL: Charles C Thomas; 1956.

4. Banks AR. A rationale for prolotherapy. J Orthopaedic Med 1991;13:54-9.

5. Burdakov D, Jensen LT, Alexopoulos H, et al. Tandempore $\mathrm{K}+$ channels mediate inhibition of orexin neurons by glucose. Neuron 2006;50:711-22.

6. Lyftogt J. Subcutaneous prolotherapy treatment of refractory knee, shoulder, and lateral elbow pain. Australasian Musculoskeletal Med 2007;12:110-2.

7. Maniquis-Smigel L, Dean Reeves K, Jeffrey Rosen H, et al. Short term analgesic effects of 5\% dextrose epidural injections for chronic low back pain: a randomized controlled trial. Anesth Pain Med 2016;7:e42550.

8. Wu YT, Ho TY, Chou YC, et al. Six-month efficacy of perineural dextrose for carpal tunnel syndrome: a prospective, randomized, double-blind, controlled trial. Mayo Clin Proc 2017;92:1179-89.

9. Yelland MJ, Sweeting KR, Lyftogt JA, Ng SK, Scuffham PA, Evans KA. Prolotherapy injections and eccentric loading exercises for painful Achilles tendinosis: a randomised trial. Br J Sports Med 2011;45:421-8.

10. Rabago D, Nourani B. Prolotherapy for osteoarthritis and tendinopathy: a descriptive review. Curr Rheumatol Rep 2017;19:34.

11. Hassan F, Trebinjac S, Murrell WD, Maffulli N. The effectiveness of prolotherapy in treating knee osteoarthritis in adults: a systematic review. Br Med Bull 2017;122:91108.

12. Rabago D, Patterson JJ, Mundt $M$, et al. Dextrose prolotherapy for knee osteoarthritis: a randomized controlled trial. Ann Fam Med 2013;11:229-37.

13. Sit RW, Wu RW, Reeves KD, et al. Efficacy of intra-articular hypertonic dextrose prolotherapy versus normal saline for knee osteoarthritis: a protocol for a triple-blinded randomized controlled trial. BMC Complement Altern Med 2018;18:157.

14. Saltzman BM, Leroux T, Meyer MA, et al. The therapeutic effect of intra-articular normal saline injections for knee osteoarthritis: a meta-analysis of evidence level 1 studies. Am J Sports Med 2017;45:2647-53.

15. Lund I, Lundeberg T. Are minimal, superficial or sham acupuncture procedures acceptable as inert placebo controls? Acupunct Med 2006;24:13-5.

16. Dworkin RH, Turk DC, Farrar JT, et al. Core outcome measures for chronic pain clinical trials: IMMPACT recommendations. Pain 2005;113:9-19. 